\title{
Fibrous dysplasia of proximal humerus - A case report
}

\author{
Vikramjit Singh $^{1, *}$, Arvind Kumar ${ }^{2}$, Sudhir Rawat ${ }^{3}$, Parth Deshmukh ${ }^{4}$, Anirudh Bansal ${ }^{5}$ \\ $1,53^{\text {rd }}$ Year Resident, ${ }^{2}$ Associate Professor, ${ }^{3}$ Assistant Professor, ${ }^{4}$ Senior Resident, Dept. of Orthopaedics, SBKSMIRC \& Dhiraj \\ Hospital, Vadodara, Gujarat, India
}

*Corresponding Author:

Email: vikramjit_singh91@y ahoo.com

\begin{abstract}
Fibrous dy splasia is a rare disorder characterized by replacement of normal tissue with fibrous connective tissue. It mostly occurs in craniofacial bones, femur, tibia and pelvis. It can also be associated with various endocrine conditions. Symptoms range from pain and restriction of movements to occurrence of pathological fractures. This is case report of 52 years old male patient with fibrous dysplasia in proximal humerus presenting with complaints of pain and restriction of movements around the shoulder treated with surgical curettage, chemical cauterization and bone grafting with internal fixation.
\end{abstract}

Keywords: Fibrous dy splasia, Pathological fracture, Internal fixation, Curettage, Humerus.

\section{Introduction}

Fibrous dysplasia is a skeletal disorder in which normal bone and marrow is replaced by abnormal fibrous and connective tissue leading to weakness of a bone and pathological fracture. The condition was first described by Lichtenstein and Jaffe in 1942 so the condition is many times is also referred as LictentsteinJaffe disease. Fibrous dysplasia constitute about 3-7\% of all bone tumors. Proliferation of fibrous tissue may lead to complications such as pain, deformity, fracture and functional impairment. It can affect one bone (monostotic), one limb (monomelic) or many bones (polystotic) which may occur along with café-au lait spots, macules and various endocrinopathies which is termed as Mc cune Albright syndrome. Yellowish or brownish non elevated patches of cutaneous pigmentation (café-au-lait spots), situated predominantly on the patient's trunk are co - existent findings in one-third to half of the patients affected with the polystotic disease. Fibrous dysplasia has also been reported with other endocrine dysfunctions such as hyperthyroidism, hyper parathyroidism, cushing's disease, diabetes mellitus etc. The most common sites of involvement are the cranio facial bones, femur, tibia, pelvis and sometimes humerus also. It is known to occur because of mutation in the GNAS gene. The most common presenting factors in patients with fibrous dysplasia include pain, deformities and pathological fractures. Fibrous dysplasia may rarely undergo malignant transformation this ominous change is reflected clinically with increase in pain or subsequent enlargement of the soft tissue mass. Rdilogically malignant transformation maybe confirmed with presence or appearance of a lytic areas, marked periosteal reaction and general cortical disruption with a soft tissue mass. The most common malignant transformation is osteosarcoma followed followed by fibrosarcoma and chondrosarcoma.

\section{Case Report}

A 55 year old male patient came to out patient department of our hospital with the chief complaint of pain in proximal humerus and restriction of movements around right shoulder. The patient gave no history of trauma or any form of injury. Slight pain persisted at rest which was increased during range of motion of shoulder. Laboratory investigations were normal and did not reveal any abnormality. On plain x-ray lytic area was observed in the proximal shaft of humerus.



Fig. 1:

\section{On MRI -}

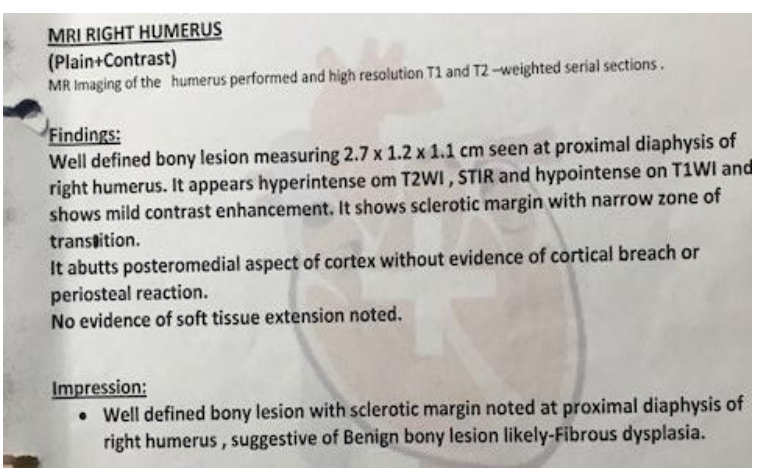

Fig. 2: 


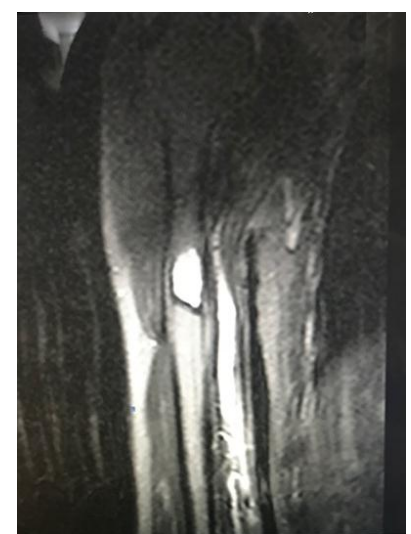

Fig. 3:

The patient was planned for surgery. Surgery was done in the form of curettage, chemical cauterization and bone grafting of lesion along with internal fixation with plating. Surgery was performed under standard protocol through anterolateral approach. Incision was closed in layers. Shoulder manipulation for stiff shoulder was also done intra operatively after fixation. Post operative recovery was well and gradual mobilization was started at first follow up at 3 weeks. Follow up were done at regular interval.

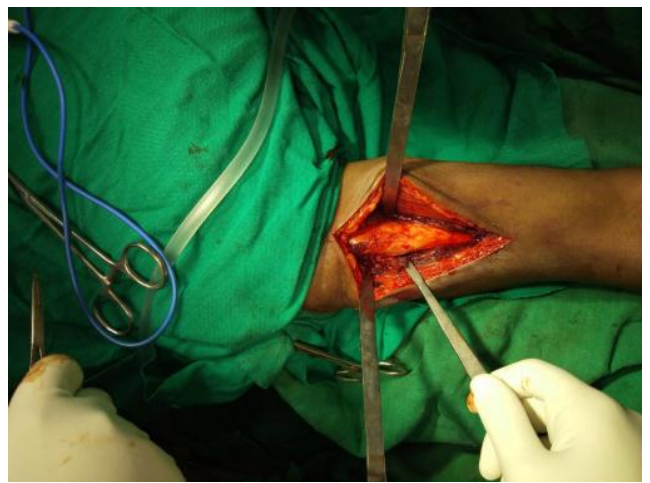

Fig. 4:

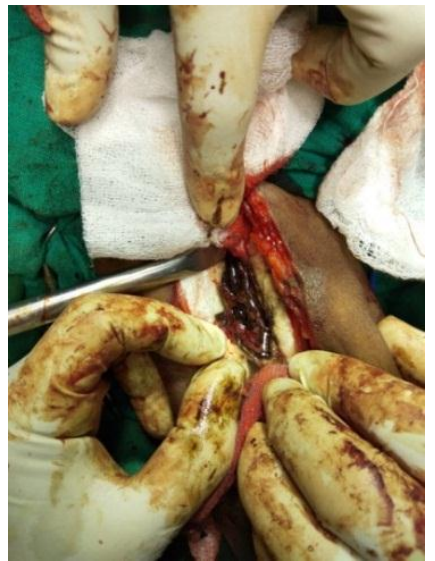

Fig. 5:

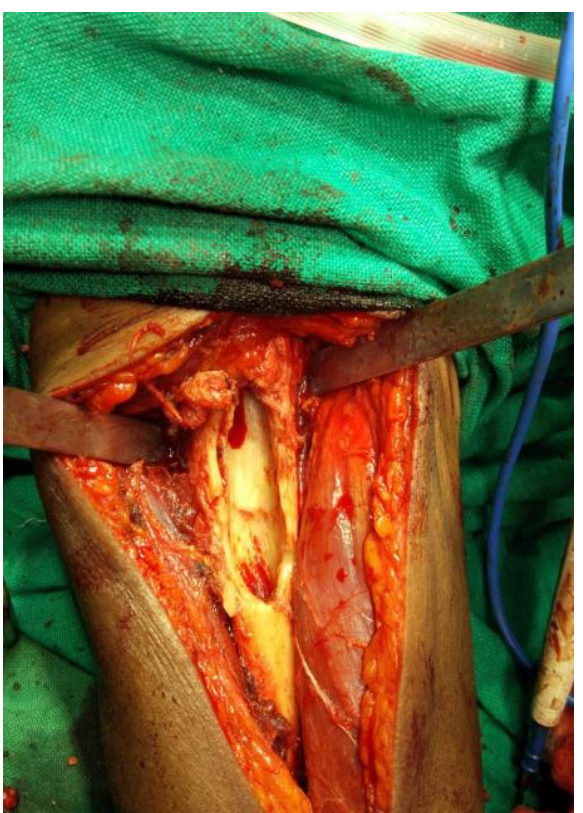

Fig. 6:

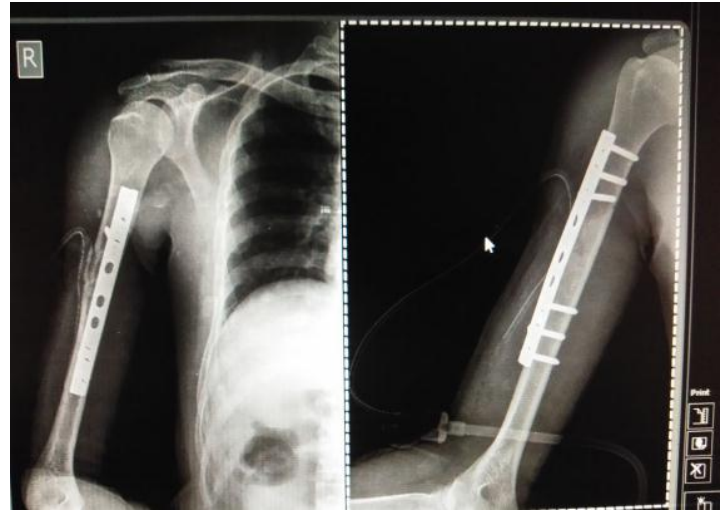

Fig. 7:

Immediate post operative $x$ ray

Histopathology reports confirmed the provisional diagnosis of fibrous dysplasia. 


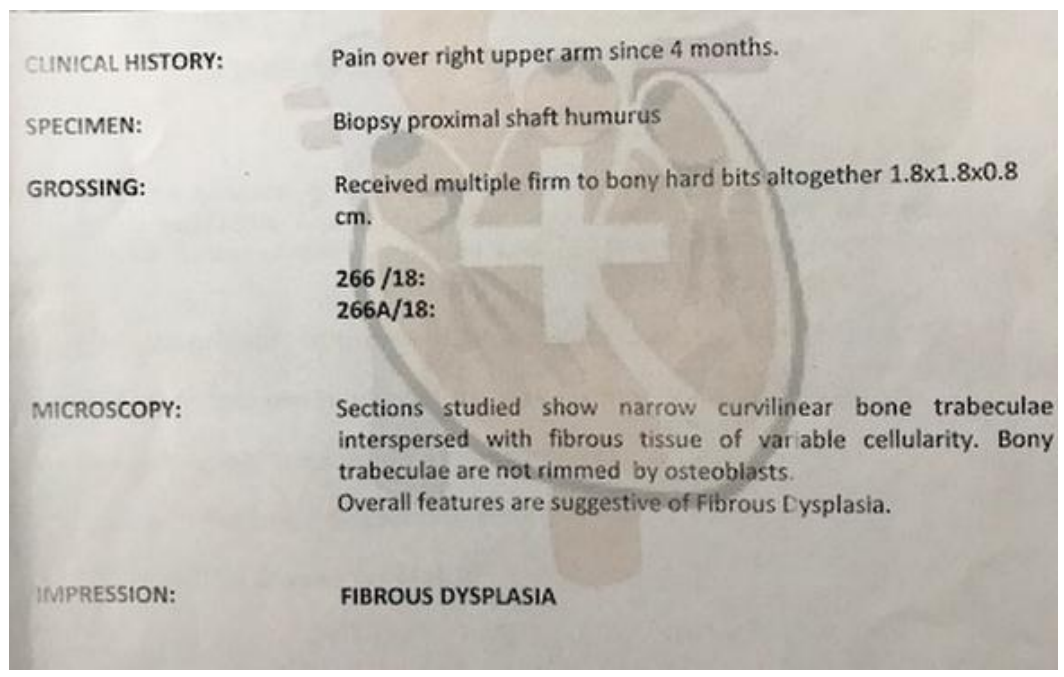

Fig. 8:

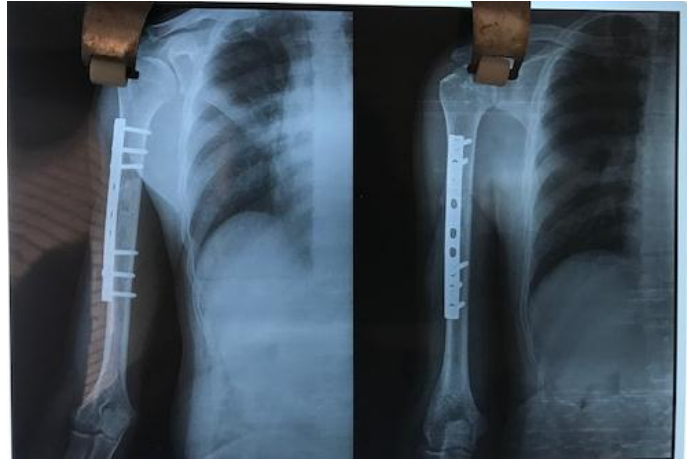

Fig. 9: 3 Months follow up

\section{Discussion and Conclusion}

Fibrous dysplasia is an abnormal growth pattern characterized by fibro- osseous replacement of mature bone marrow with immature abnormal matrix. Most lesions of osteo fibrous dysplasia affect the cortex of the bone, pre- dominantly the middle third of the diaphysis. Fibrous dysplasia constitute about 3-7\% of all bone tumours. The tissue in the lesion is immature woven bone that cannot mature into lamellar bone. This may occur due to mutation in cell surface protein. This is a somatic mutation and not a germline one. The lesion are considered to have increased number of hormone receptors which may explain why these lesions become more active during pregnancy. The condition is generally symptomless and is generally diagnosed incidentally many times due to a pathological fracture. They are sub divided into two forms i.e the monostotic form and polystotic form. Most cases of fibrous dysplasia display no particular pattern of inheritance. Monostotic lesions are generally confined to a single site and are comparatively simpler in nature and to treat. Polystotic forms usually involve multiple areas and may involve the cranio-facial bones, the ribs and the limbs. The characteristic shepherd's crook deformity in proximal femur is well documented in literature. Many times the polystotic forms are as sociated with Mc cune albright syndrome which leads to precocious puberty. The association of fibrous dysplasia with soft tissue tumors has been given the name Mazabraud's syndrome. Radiographically, fibrous dysplasia appears as a well circumscribed lesion. There is a narrow zone of transition and no periosteal reaction or soft tissue infiltration. MRI and CT scans are also useful methods of investigation. Diseases to be considered in the differential diagnosis offibrous dysplasia include chondroma, simple bone cyst, nonossifying fibromas, osteofibrous dysplasia, Paget's disease of bone, osteoblastoma, chondroblastoma, fibromyxoma of bone, adamantinoma and low-grade intramedullary osteosarcoma. Management generally includes treatment of presenting symptoms which can range from simple palliative to treatment of pathological fractures and correction of deformities including curettage, bone grafting and fixation techniques. Bisphosphonates are widely advocated for the management of this condition alongwith other supportive medications. Management of other associated conditions like endocrinopathies in polystotic form should be done and appropriately managed.

In our case report, at 6 month post operative follow up, range of movements of shoulder were full, radiological and clinical signs of union were observed by clinical and radiological examination. Our conclusion is treatment of Fibrous dysplasia with curettage, chemical cauterization and bone grafting with internal fixation (as a preventive measure to avoid pathological fracture) is an ideal treatment option.

\section{References}

1. DiCaprio MR, Enneking WF. Fibrous dysplasia. Pathophysiology, evaluation, and treatment. J Bone Joint Surg Am. 2005;87:1848-1864. doi:10.2106/JBJS.D.02942

2. Chika AT, Philomena IM. Fibrous dysplasia of the humerus: An uncommon cause of pathological fracture in a 56-year-old. Niger J Surg Res 2016;17:17-9. 
3. Stanton RP, Ippolito E, Springfield D, Lindaman L, WientroubS, Leet A. The surgical management of fibrous dysplasia of bone. Orphanet J Rare Dis 2012;7 Suppl 1:S1.

4. Fibrous dysplasia journal of the American Academy of orthopedic surgeons, number five. 2004;12:305-313.

5. Campanacci M, Laus M. Osteofibrous dysplasia of the tibia and fibula. J Bone Joint Surg [Am ]. 1981;63(3):36775.

6. Hahn SB, Kim SH, Cho NH, Choi CJ, Kim BS, Kang HJ. Treatment of osteofibrous dy splasia and associated lesions. Yonsei Med J. 2007;48(3):502-10. 